\title{
SUPPORTING ONLINE MATERIAL: Structure and Energetics of the Hydronium Hydration Shells
}

Omer Markovitch and Noam Agmon

One of several motivations for calculating $\mathrm{HB}$ strengths in the hydronium solvation shells is their inherent connection with mechanisms proposed for proton mobility in liquid water. Recent mechanisms focus on the role of the A1 bond in the Grotthuss mechanism (4,6,10,11). In the "Eigen-to-Zundel-to-Eigen" (EZE) model (4), cleavage of an $\mathrm{A} 1$ bond on the acceptor side converts an $\mathrm{H}_{3} \mathrm{O}^{+}$cation into $\mathrm{H}_{5} \mathrm{O}_{2}{ }^{+}$. Then formation of another $\mathrm{A} 1$ bond on the donor side completes the transformation. In the "Zundel-to-Zundel" (ZZ) model, possibly first depicted in the ab-initio molecular dynamics trajectory of Ref. (10), analogous HB cleavage/formation events drive a double-proton-transfer which converts directly one Zundel cation into another.
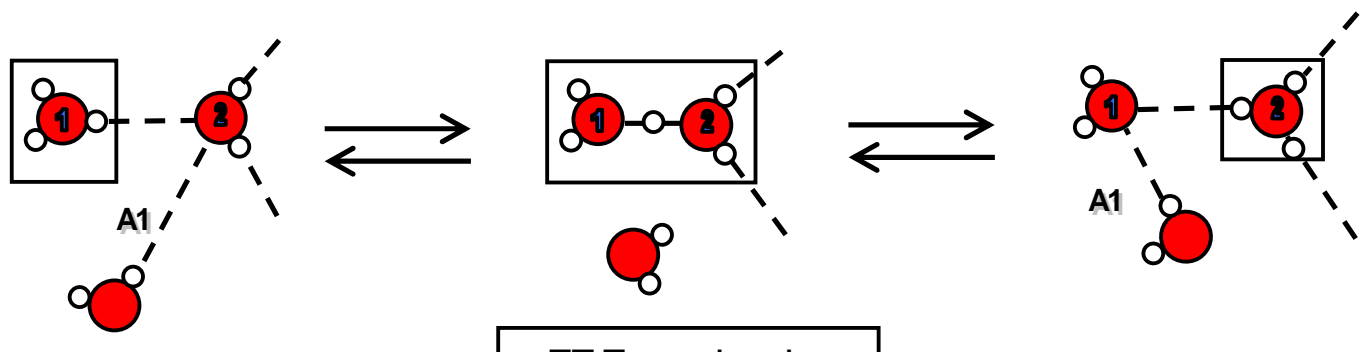

EZE mechanism
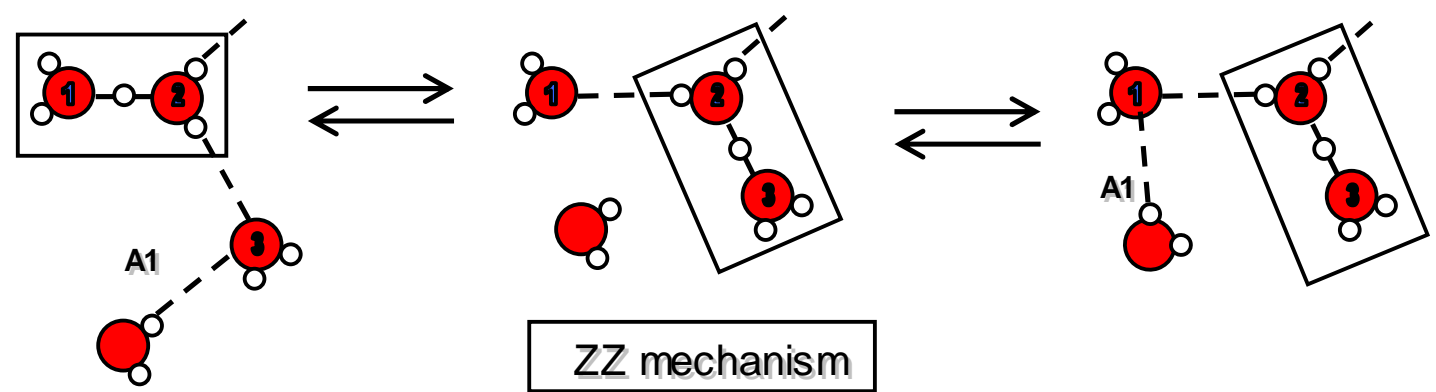

ZZ mechanism

Figure S1. Two mechanisms for proton mobility in liquid water controlled by A1 bonds. The full set of HBs are shown only for water molecule \#2. The rest should be completed by symmetry. Rectangles highlight "Eigen" vs. "Zundel” structures. 
The energies of the HBs calculated depend somewhat on the definition of a HB. In order not to introduce additional effects into the present communication we have chosen the most conventional geometrical definition using, using one distance and one angle, as shown in Fig. S2.

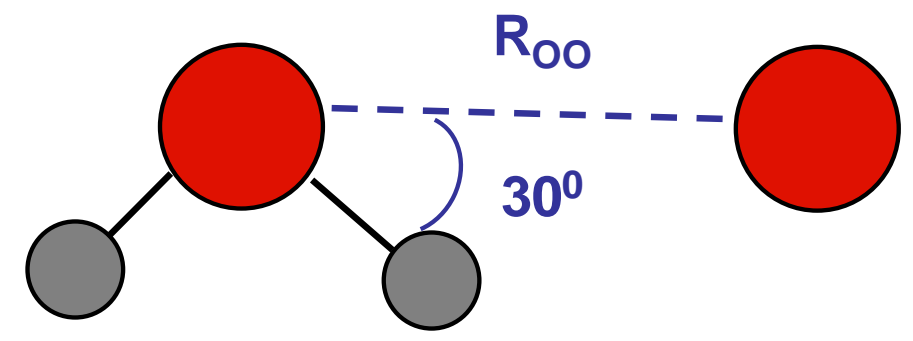

Figure S2. The cutoff distance and angle defining a HB in liquid water.

The cutoff distance, $R_{\mathrm{OO}}$, is not identical to the minimum in $g(r), R_{\min }$. This minimum is at $2.95,3.00$, and $3.35 \AA$ for $i=0,1$ and 2 , respectively. At these distances, the bond enthalpy is still sensitive to $R_{\mathrm{OO}}$. To check this, we have calculated $\Delta H^{0}$ as a function of $R_{\mathrm{OO}}$ for the different donor-type bonds (Fig. S3). A reduced set of trajectories and temperatures was used to speed up the calculation. The plots tend to a plateau at somewhat larger distances than $R_{\min }$ (red points). Interestingly, these cutoff distances are about $0.75 \AA$ larger than the distances at the peak of $g(r)$. Results reported in Tbl. 1 of the article were calculated at these cutoff distances. Incidentally, we note some (small) systematic differences between the red points and the $\Delta H^{0}$ in the table, which appear to be larger than the increased noise of the smaller sample space. Possibly, the ergodic property does not yet hold for a 250 ps trajectory, and therefore averaging the results over several trajectories with different initial conditions is required. We have used 4-10 trajectory segments at each temperature. Ai HB strengths were calculated at the cutoff determined for the corresponding Di bond. 


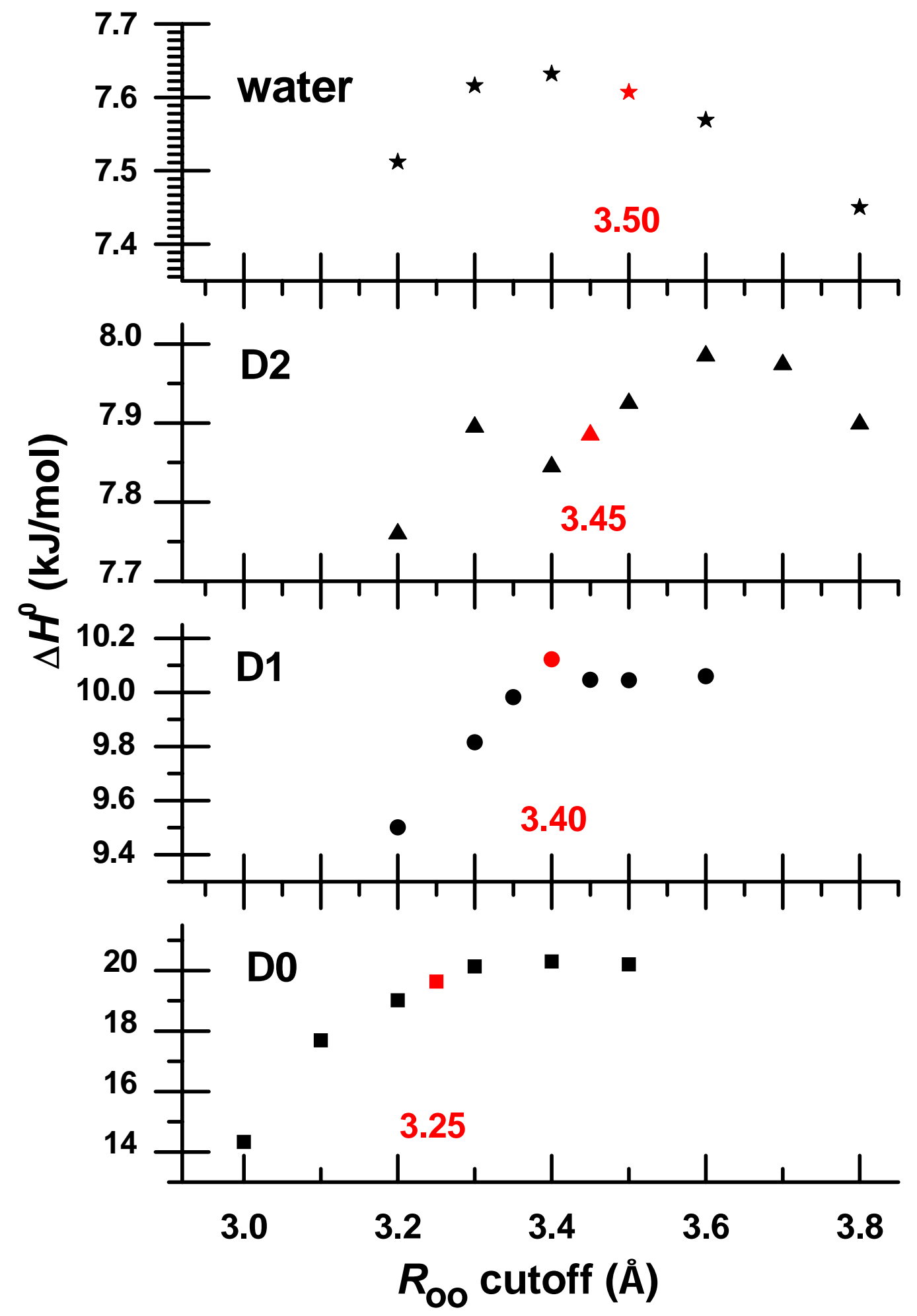

Figure S3. Determination of the cutoff distance (red) in the HB definition. 